\title{
Teashirt 3 expression in the chick embryo reveals a remarkable association with tendon development
}

\author{
Isabelle Manfroid ${ }^{1}$, Xavier Caubit, Christophe Marcelle, Laurent Fasano * \\ Institut de Biologie du Développement de Marseille-Luminy, UMR CNRS 6216, Centre National de la Recherche Scientifique-Université de la Méditerranée, \\ Campus de Luminy, F-13288 Marseille cedex 09, France
}

Received 21 December 2005; received in revised form 3 March 2006; accepted 3 March 2006

Available online 2 May 2006

\begin{abstract}
Drosophila teashirt (tsh) is involved in the patterning of the trunk identity together with the Hox genes. In addition, it is also a player in the Wingless and the Hedgehog pathways. In birds and mammals, three Tshz genes are identified and the expression patterns for mouse $T s h z 1$ and $T s h z 2$ have been reported during embryogenesis. Recently, we showed that all three mouse Tshz genes can rescue the Drosophila tsh loss-of-function phenotype, indicating that the function of the teashirt genes has been conserved during evolution. Here we describe the expression pattern of chick TSHZ3 during embryogenesis. Chick TSHZ3 is expressed in several tissues including mesodermal derivatives, the central and peripheral nervous systems. Emphasis is laid on the dynamic expression occurring in regions of the somites and limbs where tendons develop. We show that TSHZ3 is activated in the somites by FGF8, a known inducer of the tendon marker $S C X$.
\end{abstract}

(C) 2006 Elsevier B.V. All rights reserved.

Keywords: Teashirt; Chick; Mouse; Drosophila; Scleraxis; Tendon; Neurons

\section{Results and discussion}

Drosophila teashirt (tsh) encodes for a zinc finger transcription factor that is crucial for the patterning of the trunk identity in collaboration with the Hox genes (Fasano et al., 1991; Röder et al., 1992). Tsh acts also in the Wingless and the Hedgehog pathways (Angelats et al., 2002; Gallet et al., 1998, 1999). In addition, tsh function is required for the midgut morphogenesis (Mathies et al., 1994) and for the development of adult appendages (Bessa et al., 2002; Erkner et al., 1999; Pan and Rubin, 1998; Soanes et al., 2001; Wu and Cohen, 2000). In vertebrates, three teashirt ( $\left.T_{s h} z\right)$ genes have been identified in mouse

\footnotetext{
* Corresponding author. Tel.: +33 4912696 03; fax: +33 491820682 .

E-mail address: fasano@ibdml.univ-mrs.fr (L. Fasano).

${ }^{1}$ Present address: Laboratoire de Biologie Moléculaire et de Génie Génétique, Center of Biomedical Integrative Genoproteomics (CBIG), Université de Liège, Institut de Chimie, Bâtiment B6, B-4000 Liège (SartTilman), Belgium.
}

and human. Expression patterns during embryogenesis were reported for mouse $T s h z 1$ and $T s h z 2$ and are consistent with a role in trunk specification in vertebrates (Caubit et al., 2000). Recently, we tested whether $T s h z 1, T s h z 2$, or $T s h z 3$ could rescue $t s h$ loss-of-function in flies. We showed that all three mouse $T s h z$ rescued with high efficiency homeotic transformation and abnormal trunk morphogenesis, two defects observed in tsh null mutant Drosophila embryos. Rescue of Drosophila tsh null mutant by the mouse orthologs demonstrates that the function of Tshz genes is phylogenetically conserved (Manfroid et al., 2004). Here we describe the expression of the third member of the $T s h z$ genes family, chickTSHZ3, during chick embryogenesis and show a remarkable expression in tendons.

\subsection{Identification of chick Tsh genes}

In a BLAST search with the amino acid sequences of mouse $T s h z$ genes against the chick draft genome database (Ensembl Genome Browser (currently v.36-Dec2005), 
http://www.ensembl.org/Gallus_gallus/index.html), we found three genes. The sequences show high similarity to the tsh/tio family. Alignment of these sequences revealed characteristic amino acids thereby unequivocally identifying the three $T s h z$ genes of vertebrates (data not shown). Phylogenetic analyses based on the protein sequences using Neighbor-joining method clearly groups chick TSHZ3 with other vertebrate $T s h z 3$ sequences (Fig. 1). Based on these results, we named the new genes (Chick)TSHZ1, (Chick)TSHZ2 and (Chick)TSHZ3. Chromosomal locations of (Chick) TSHZ genes have been identified. TSHZ1, $T S H Z 2$ and TSHZ3 are located on chromosome 2, 20 and 11 , respectively.

\subsection{Overall TSHZ3 expression during early chick embryogenesis}

We could not detect TSHZ3 mRNAs by in situ hybridization prior to $\mathrm{HH}$ stage 10 when a faint expression takes place in the neural plate (not shown). Between $\mathrm{HH}$ stage 10 and 15, additional sites of expression are observed. TSHZ3 demarcates the neural tube, the lateral mesoderm (Fig. 2A) and the region of the foregut (Fig. 2B). Rostrally, rhombomere $\mathrm{r} 4$, anterior to the otic vesicle, constitutes the anterior limit of expression in the neural tube (Fig. 2B). At HH stage 21 (Figs. 2C and D), TSHZ3 is found in the mesenchyme of the posterior aspect of the limb buds, in branchial arches (BA) posterior to BA I, at the level of the foregut and in the lateral mesoderm between the fore- and the hindlimb buds. The most striking expression is observed in the somites. The expression becomes detectable in the posterior part by $\mathrm{HH}$ stage 18, and intensifies as development proceeds (Figs. 2C and D). No or very weak TSHZ3 expression is observed in the four most anterior somites (occipital somites, Fig. 2D). The staining in the head, not reproducible, is likely due to the trapping of the probe/substrate. Around HH stage 24 (Fig. 2E), TSHZ3 appears in the anterior part of the somites in addition to the posterior domain of expression. We focused our analysis on this interesting expression.
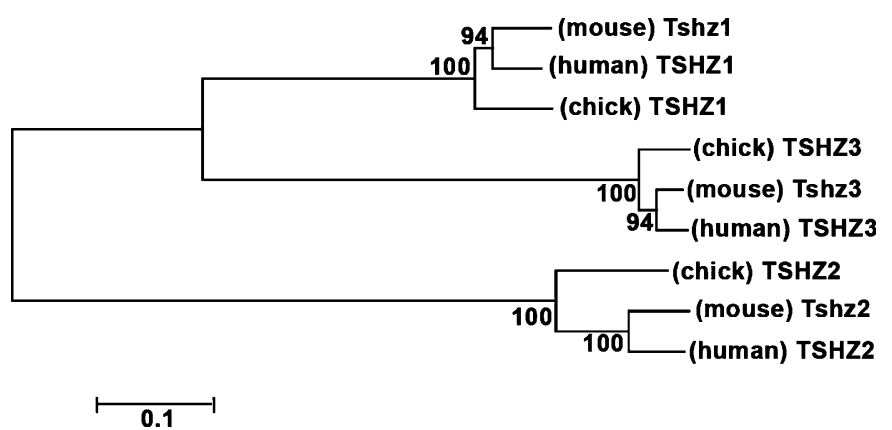

Fig. 1. Phylogenetic tree of Tshz sequences. Tshz proteins fall into three classes. The numbers of the interior branches refer to the bootstrap values with 100 replicates.

\subsection{Expression of TSHZ3 in the forming tendons}

A dorsal view of the trunk at HH stage 27 uncovers the similarity between $T S H Z 3$ and Scleraxis $(S C X$ ) expression in the somites (Figs. 2F and G). $S C X$ marks the syndetome, a somitic compartment formed by the tendon progenitors localized at the anterior and posterior margins of the somites (Brent et al., 2003; Schweitzer et al., 2001). Transverse sections (Figs. $2 \mathrm{H}$ and I) show that TSHZ3 and $S C X$ both delineate the same region - a narrow stripe of mesenchyme underlying the myotome. However, frontal sections reveal that the TSHZ3 domain is broader than the thin, V-shaped $S C X$ expression domain (Figs. 2J and K). TSHZ3 and $S C X$ do not overlap with the myofibers immunostained with the anti-myosin heavy chain MF-20 antibody. Surprisingly, in slightly younger embryos (HH stage 24), $T S H Z 3$ and $S C X$ match more remarkably (Figs. $2 \mathrm{~L}$ and M). Thus, TSHZ3 expression in the somites follows that of $S C X$ (Brent et al., 2003; Brent and Tabin, 2004), first in the same domain as SCX (HH stage 24), and subsequently in a broader domain (HH stage 27).

We also examined the expression in the limbs. At $\mathrm{HH}$ stage 23, TSHZ3 labels the posterior and anterior part of the hindlimb bud (Fig. 2N). TSHZ3 transcripts are similarly distributed in the forelimb bud (not shown). These expression domains are distinct from the area defined by the tendons progenitors and the forming muscles, since, at this stage, both cell types occupy the central region of the limb bud (Schweitzer et al., 2001). On sections, TSHZ3 is separate from $T C F 4$, which is intimately associated with forming limb muscles and tendons (Figs. $2 \mathrm{O}$ and $\mathrm{P}$, Kardon et al., 2003). By HH stage 27, TSHZ3 expression pattern becomes more complex (Fig. 2Q) and partial overlapping appears between TSHZ3 and SCX (Figs. 2R and S). In older embryos, TSHZ3 displays the most pronounced staining in the myotendinous junctions, which are also strongly marked by $S C X$ (HH stage 34, Figs. 2T and U). Astonishingly, while early TSHZ3 expression is excluded from the myotome and from the limb muscles, subsequent $T S H Z 3$ transcription is visible in muscles and in surrounding connective tissues at $\mathrm{HH}$ stage 34 . Thus, $T S H Z 3$ marks broader domains than $S C X$ at later stages.

It has been shown that $S C X$ expression is induced by myotomal FGFs (Brent et al., 2003). Here we show that, as demonstrated for $S C X$, insertion of FGF8-coated beads in the trunk somites results in a faint but reproducible ectopic TSHZ3 expression after $24 \mathrm{~h}$. This upregulation occurs in cells surrounding the beads that normally do not express TSHZ3 nor SCX (HH stage 26, Figs. 2V and W). Enhanced TSHZ3 transcription is also detected upon shorter treatments with FGF8 (after $12 \mathrm{~h}$, not shown).

\subsection{Other places of TSHZ3 expression in the chick embryo}

In the neural tube, we noticed dissimilarity between TSHZ3 expression at brachial and lumbar positions. At 

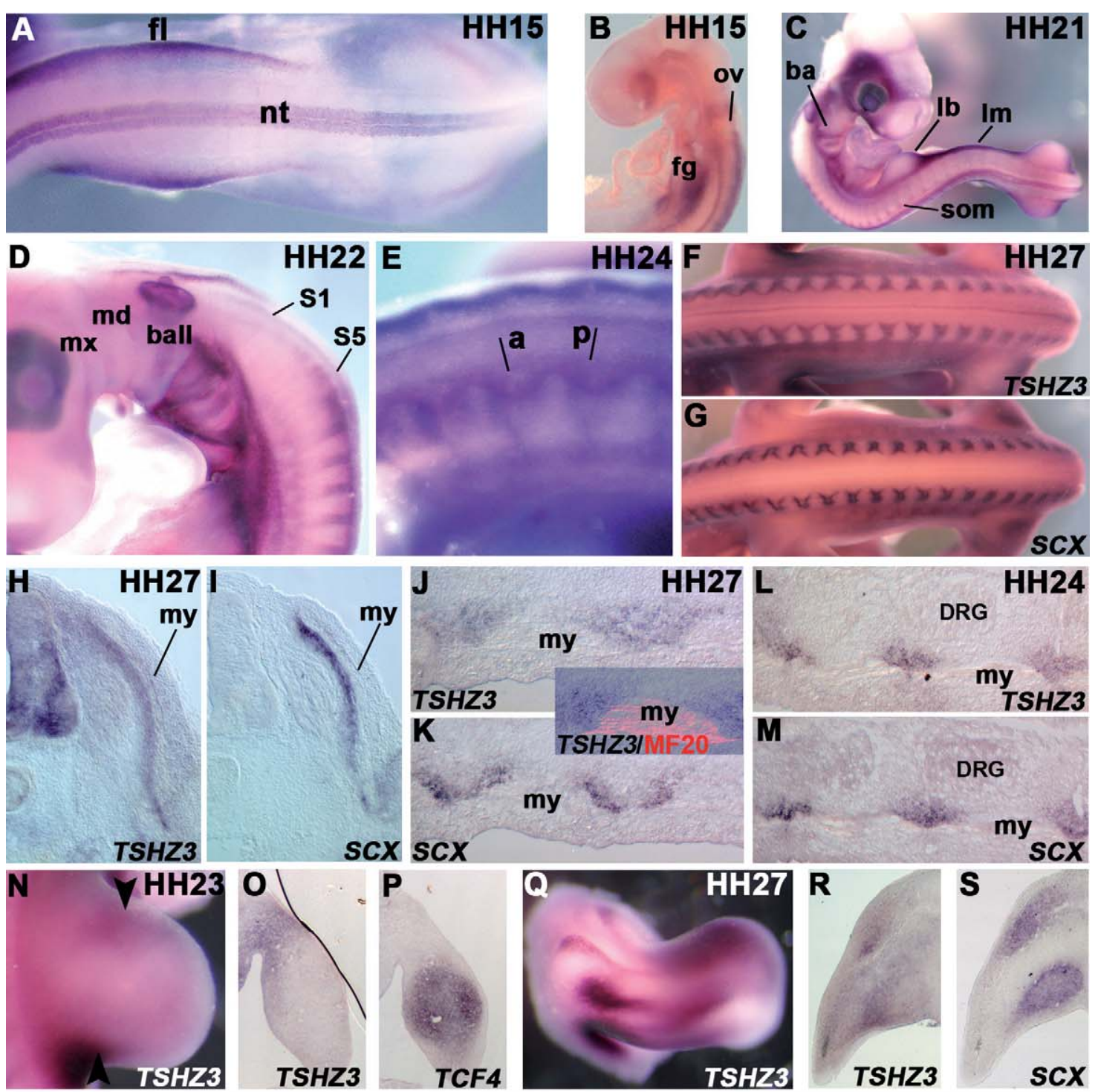

HH24
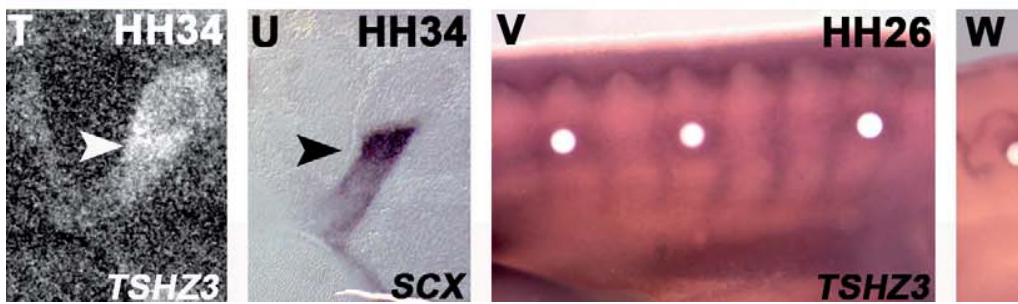

DRG

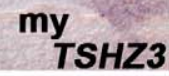

TSHZ3

my $\operatorname{scX}$

Fig. 2. Expression of TSHZ3 in the chick embryo. (A and B) Whole mount in situ hybridizations at HH stage 15. (C) Additional sites of expression at HH stage 21. (D) Close-up of the branchial arches and the anterior somites at HH stage 22. (E) Close-up, at HH stage 24, of the brachial somites (a: anterior, p: posterior parts of the somites). (F and G) TSHZ3 (F) and $S C X(\mathrm{G})$ expression in the trunk at HH stage 27 (dorsal view). (H and I) Transverse adjacent sections at the brachial level hybridized with $T S H Z 3(\mathrm{H})$ and $S C X(\mathrm{I})$ probes at $\mathrm{HH}$ stage 27. (J and K) Frontal sections at the same stage and same A/P axis level hybridized with TSHZ3 $(\mathrm{J})$ and $S C X(\mathrm{~K})$ probes. The inset shows TSHZ3 and MF20. (L and M) Transverse sections at the brachial level through a HH stage 24 embryo. (N and Q) Whole mount TSHZ3 expression in a hindlimb bud at HH stage $23(\mathrm{~N})$, and in a HH stage 27 forelimb (Q). The arrowheads point out the TSHZ3-expressing posterior and anterior regions. (O, P-R, and S) Parasagittal transverse sections through a HH stage 23 hindlimb $(\mathrm{O}$ and $\mathrm{P})$ and $\mathrm{HH}$ stage 27 forelimb (R and $\mathrm{S}$ ) stained for TSHZ3 (O and $\mathrm{R})$ and TCF4 (P and $\mathrm{S})$. (T and $\mathrm{U})$ Section through the thigh at HH stage 34 hybridized with ${ }^{35}$ S-labelled $T S H Z 3(\mathrm{~T})$ probe or DIG-SCX (U). Arrowheads designate the robust expression in the myotendinous junction. (V and W) Up-regulated $T S H Z 3(\mathrm{~V})$ and $S C X(\mathrm{~W})$ expression around FGF8-coated beads. DRG: dorsal root ganglia, fg: foregut, fl: forelimb bud, lb: limb bud, lm: lateral mesoderm, my: myotome, ov: otic vesicle, som: somite, nt: neural tube. Whole mount and frontal sections, except in (B): anterior to the left. Transverse and sagittal sections: dorsal to the top.

the brachial level of a HH stage 21 embryo, TSHZ3 demarcates a ventral region located dorsal to the floor plate comprising motor neurons progenitors. TSHZ3 expression is also detected in lateral regions of the spinal cord in the marginal zone (Fig. 3A, arrowhead). Later, at HH stage 34 , the dorsal half of the spinal cord displays robust expres- 


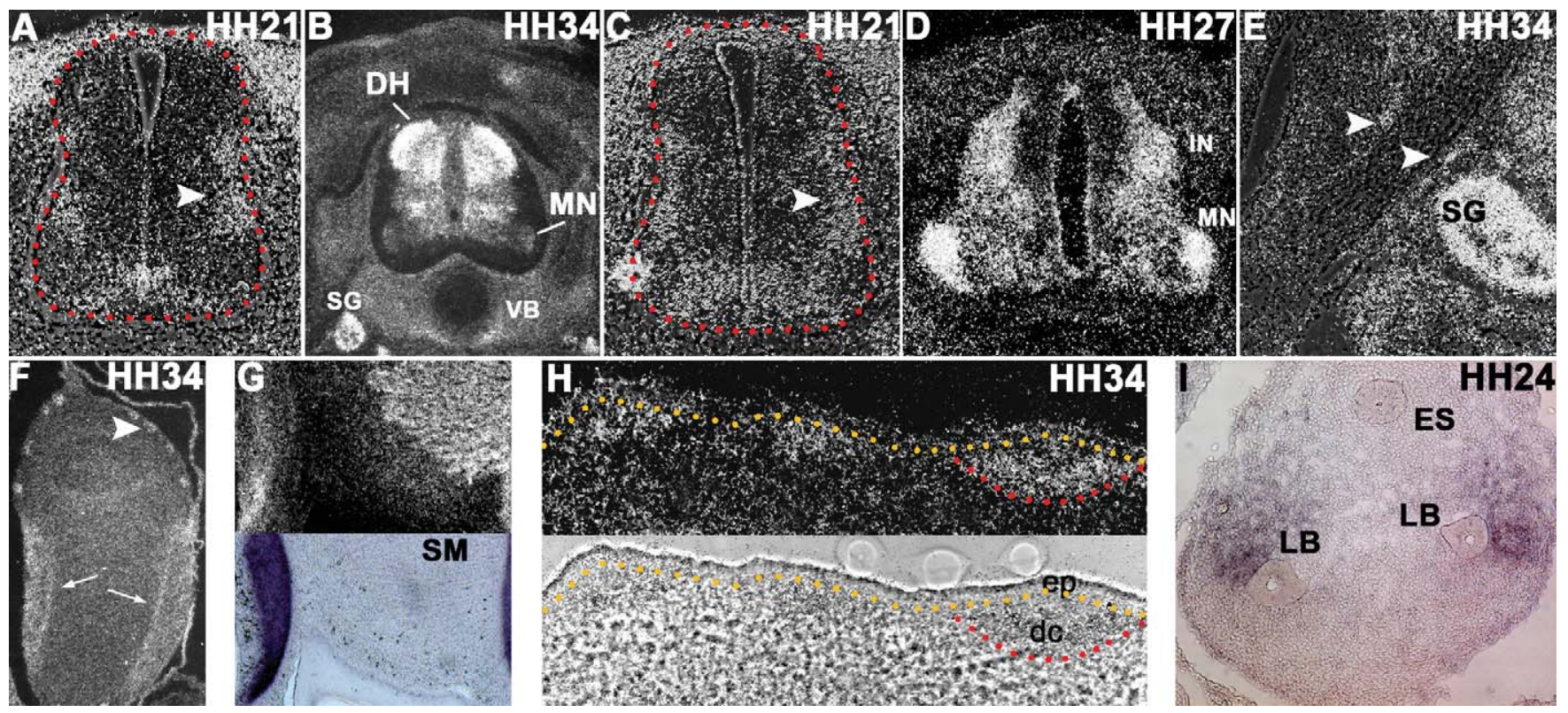

Fig. 3. Other places of TSHZ3 expression. (A and C) ${ }^{35}$ S-labelled TSHZ3 transverse sections through the neural tube at HH stage 21 at brachial (A) and lumbar (C) level. Arrowheads point to the lateral marginal zone. Red dotted lines delineate the spinal cord. (B and D) Same as (A and C) at HH stage 34 (B) and 27 (D). (E) Axonal tract arising from the spinal cord at HH stage 34. The arrowheads indicate TSHZ3-expressing cells at the border. ( $\mathrm{F}$ and $\mathrm{G}$ ) Two sections through the HH stage 34 gizzard labelled with TSHZ3 (F and G, top) and $S C X$ (G, bottom) probes. The arrows indicate the laminar tendon and the arrowhead point to one of the forming enteric nervous systems elements (eight ganglia are observed on this section). (H) Transverse section through the skin. The ectoderm is apparent in bright field (demarcated by a yellow dotted line). The red line underlines the condensing mesenchyme (dermal condensates) beneath the ectodermal placode of the feather bud. (I) Zone of the esophagus (dorsal) and of the two lung buds (ventral). Photographs (A, C, E) are bright field/black field composite images. DH: dorsal horn, dc: dermal condensate, ep: ectodermal placode, ES: esophagus, IN: interneurons, LB: lung bud, MN: motoneurons, SG: sympathetic ganglia, SM: smooth muscle, VB: vertebral body.

sion; the TSHZ3 positive domain covers the subventricular zone and the dorsal horn (Fig. 3B). A very weak staining is also observed in a lateral population of motor neurons. At the lumbar level of HH stage 21 embryos, TSHZ3 exhibits expanded ventral expression compared to the brachial level (Fig. 3C). TSHZ3 is found in the marginal zone as well (arrowhead). Later, in contrast to the brachial level, sustained expression is encountered in motor neurons (Fig. 3D). Dorsally, TSHZ3 is prominently expressed in the mantle layer indicating expression in the alar plate interneurons. In addition to the central nervous system, TSHZ3 is also expressed in the peripheral nervous system. Notable examples are the sympathetic ganglia (Figs. 3B and E), cells along the axonal tracts where the Schwann cells develop (Fig. 3E) and the enteric nervous system. In the latter, strong TSHZ3 expression is observed in aggregates of cells within the outer gut mesenchyme of the gizzard constituting the ganglia of the myenteric plexus (Fig. 3F, arrowhead). In addition, consistent with TSHZ3 expression in tendons, TSHZ3 is found in the laminar tendon of the gizzard (compare with $S C X$ in Figs. $3 \mathrm{~F}$ and G). Smooth muscles also express TSHZ3 (Fig. 3G, and in the intestine, not shown).

In addition, TSHZ3 is detected in zones of condensing mesenchyme forming the cartilage (see the vertebral body encircling the notochord in Fig. 3B). TSHZ3 expression is also detected in several tissues where epithelio-mesenchymal interactions take place, such as the feather anlagens (HH stage 34, Fig. 3H, also evident in Fig. 3B) and in the mesenchyme in the area of the lung buds (HH stage 24, Fig. 3I).

TSHZ3, like Tshz1 and Tshz2 in mouse, is expressed in the nervous system and in mesodermal derivatives. A noteworthy feature of $T S H Z 3$ is its expression in developing tendons and exclusion from the forming muscles at early stages. Later, TSHZ3 displays enlarged expression in the myotendinous junctions, the muscles (skeletal and smooth muscles) and connective tissues. We hope this study will improve our knowledge of the $T s h z$ genes expression patterns for understanding their specific and redundant functions.

\section{Experimental procedures}

\subsection{Identification of TSHZ3 clones and phylogenetic reconstruction}

In a BLAST search with the amino acid sequences of mouse $T s h z$ genes we identified a chick EST clone presenting a high degree of homology with the mouse and human $T s h z 3$ genes (C482, kind gift of Dr. Nat Bumstead). C482 was used to identify a longest chick EST clone, ChEST257k10 (ID BU471594). This clone was used to generate TSHZ3 riboprobe. Sequence alignments were analyzed by Neighbor-joining (NJ) (Gamma model of distances and sites pairwise deletion) with MEGA version 3.0 (Kumar et al., 2004). Confidence estimates included bootstrap analysis with 100 replicates. 


\subsection{Processing of the tissues}

Fertilized chicken eggs were purchased from a commercial source. Eggs were routinely incubated, opened and staged according to Hamburger and Hamilton (1951). The specimens were fixed in 4\% paraformaldehyde and processed for whole-mount in situ hybridization or cryopreserved in 30\% sucrose and embedded in OCT (Tissue-Tek) for freezing and sectioned at $10-15 \mu \mathrm{m}$ on the cryostat for tissue section in situ hybridization and immunodetection.

\subsection{Chick TSHZ3 and SCX probes and in situ hybridizations}

Whole mount and on sections in situ hybridizations were performed using digoxigenin (Boehringer)-labelled chick TSHZ3 (DNA linearization: NotI, antisense RNA synthesis: T3), Scleraxis (SCX) (Schweitzer et al., 2001; DNA linearization: EcoRI, antisense RNA synthesis: T3), and TCF4 (Kardon et al., 2003) riboprobes according to Henrique et al. (1995). Embryos were photographed using a Leica MZ8 dissecting microscope with a Canon D30 colour digital camera. Zeiss Axiophot2 microscope equipped with a Nikon DXM1200 Digital Camera. The automatic camera tamer software (ACT-1 Version 2.10, Nikon Corporation) was used to allow operation of the Digital Camera Control unit from a networked high-performance PC. The $S C X$ cDNA is a kind gift of D. Duprez.

Radioactive in situ hybridizations with ${ }^{35}$ S-labelled TSHZ3 riboprobe on sections were performed as described in Caubit et al. (2005).

\subsection{Immunohistochemistry staining procedure}

Immunodetection of the myosin heavy chain was performed on cryosections of embryos previously processed for TSHZ3 whole mount in situ hybridization. 1:20 dilution of an MF-20 hybridoma supernatant directed against the embryonicmyosin heavy chain (Developmental Studies Hybridoma Bank) and was detected by Alexa 546 fluorophor-labelled secondary antibodies (Jackson).

\subsection{FGF8-soaked beads procedure}

Heparin-immobilized acrylic beads (Sigma) were saturated overnight at $4{ }^{\circ} \mathrm{C}$ in a solution of $1 \mu \mathrm{g} / \mu \mathrm{l}$ of FGF8 (R\&D systems) diluted in PBS $0.2 \%$ BSA. Beads were then implanted in the interlimb somites of a $\mathrm{HH}$ stage 23 embryos $24 \mathrm{~h}$ prior to dissection.

\section{Acknowledgements}

We thank Dr. D. Duprez for providing us with the $S C X$ and TCF4 probes and Dr. Y. Perez for the phylogenetic analysis. We are grateful to Lois J. Maltais and the Mouse Genome Nomenclature Committee (MGNC) and the HUGO Gene Nomenclature Committee (HGNC) that have approved the nomenclature for the teashirt genes family. We are grateful to M.C. Delphini and members of the Fasano's lab for critical reading of the manuscript. We also wish to thank the members of C. Marcelle's lab for their technical assistance and G. Gabella for the analysis in the gizzard. This work was supported by "l'Association Française contre les Myopathies" (A.F.M.) in contract with F.L.I. Manfroid was a fellow of the A.F.M.

\section{References}

Angelats, C., Gallet, A., Therond, P., Fasano, L., Kerridge, S., 2002. Cubitus interruptus acts to specify naked cuticle in the trunk of Drosophila embryos. Dev. Biol. 241, 132-144.
Bessa, J., Gebelein, B., Pichaud, F., Casares, F., Mann, R.S., 2002. Combinatorial control of Drosophila eye development by eyeless, homothorax, and teashirt. Genes Dev. 16, 2415-2427.

Brent, A.E., Schweitzer, R., Tabin, C.J., 2003. A somitic compartment of tendon progenitors. Cell 113, 235-248.

Brent, A.E., Tabin, C.J., 2004. FGF acts directly on the somitic tendon progenitors through the Ets transcription factors Pea3 and Erm to regulate scleraxis expression. Development 131, 3885-3896.

Caubit, X., Core, N., Boned, A., Kerridge, S., Djabali, M., Fasano, L., 2000. Vertebrate orthologues of the Drosophila region-specific patterning gene teashirt. Mech. Dev. 91, 445-448.

Caubit, X., Tiveron, M.C., Cremer, H., Fasano, L., 2005. Expression patterns of the three Teashirt-related genes define specific boundaries in the developing and postnatal mouse forebrain. J. Comp. Neurol. 486, 76-88.

Erkner, A., Gallet, A., Angelats, C., Fasano, L., Kerridge, S., 1999. The role of Teashirt in proximal leg development in Drosophila: ectopic Teashirt expression reveals different cell behaviours in ventral and dorsal domains. Dev. Biol. 215, 221-232.

Fasano, L., Roder, L., Core, N., Alexandre, E., Vola, C., Jacq, B., Kerridge, S., 1991. The gene teashirt is required for the development of Drosophila embryonic trunk segments and encodes a protein with widely spaced zinc finger motifs. Cell 64, 63-79.

Gallet, A., Angelats, C., Erkner, A., Charroux, B., Fasano, L., Kerridge, S., 1999. The C-terminal domain of armadillo binds to hypophosphorylated teashirt to modulate wingless signalling in Drosophila. EMBO J. 18, 2208-2217.

Gallet, A., Erkner, A., Charroux, B., Fasano, L., Kerridge, S., 1998. Trunk-specific modulation of wingless signalling in Drosophila by teashirt binding to armadillo. Curr. Biol. 8, 893-902.

Hamburger, V., Hamilton, H., 1951. A series of normal stages in the development of the chick embryo. J. Morphol. 88, 49-92.

Henrique, D., Adam, J., Myat, A., Chitnis, A., Lewis, J., Ish-Horowicz, D., 1995. Expression of a Delta homologue in prospective neurons in the chick. Nature 375, 787-790.

Kardon, G., Harfe, B.D., Tabin, C.J., 2003. A Tcf4-positive mesodermal population provides a prepattern for vertebrate limb muscle patterning. Dev. Cell 5, 937-944.

Kumar, S., Tamura, K., Nei, M., 2004. MEGA3: Integrated software for molecular evolutionary genetics analysis and sequence alignment. Brief. Bioinformatics 5, 150-163.

Manfroid, I., Caubit, X., Kerridge, S., Fasano, L., 2004. Three putative murine Teashirt orthologues specify trunk structures in Drosophila in the same way as the Drosophila teashirt gene. Development 131, 1065-1073.

Mathies, L.D., Kerridge, S., Scott, M.P., 1994. Role of the teashirt gene in Drosophila midgut morphogenesis: secreted proteins mediate the action of homeotic genes. Development 120, 2799-2809.

Pan, D., Rubin, G.M., 1998. Targeted expression of teashirt induces ectopic eyes in Drosophila. Proc. Natl. Acad. Sci. USA 95, $15508-15512$.

Röder, L., Vola, C., Kerridge, S., 1992. The role of the teashirt gene in trunk segmental identity in Drosophila. Development 115, 1017-1033.

Schweitzer, R., Chyung, J.H., Murtaugh, L.C., Brent, A.E., Rosen, V., Olson, E.N., Lassar, A., Tabin, C.J., 2001. Analysis of the tendon cell fate using Scleraxis, a specific marker for tendons and ligaments. Development 128, 3855-3866.

Soanes, K.H., MacKay, J.O., Core, N., Heslip, T., Kerridge, S., Bell, J.B., 2001. Identification of a regulatory allele of teashirt (tsh) in Drosophila melanogaster that affects wing hinge development. An adult-specific tsh enhancer in Drosophila. Mech Dev. 105, 145-151.

Wu, J., Cohen, S.M., 2000. Proximal distal axis formation in the Drosophila leg: distinct functions of teashirt and homothorax in the proximal leg. Mech Dev. 94, 47-56. 